\title{
Increasing Dietetic Referrals: Perceptions of general practitioners, practice nurses and dietitians
}

\author{
Lana J. MITCHELL, ${ }^{1}$ Lesley MACDONALD-WICKS ${ }^{1}$ and Sandra CAPRA ${ }^{2}$ \\ ${ }^{1}$ Faculty of Health, School of Health Sciences, University of Newcastle, Callaghan, New \\ South Wales, and \\ ${ }^{2} \mathrm{UQ}$ Centre for Clinical Research, The University of Queensland, Brisbane, \\ Queensland, Australia
}

\section{ABSTRACT}

Aim: to examine Australian private practice dietitians' relationships with general practitioners and practice nurses as a factor that influences dietetic referrals.

Methods: Semi-structured telephone interviews and an online survey were conducted with Australian private practice dietitians ( $\mathrm{n}=52$ and $\mathrm{n}=90$ respectively) between April and May 2008. Semi-structured questionnaires were conducted with general practitioners $(n=11)$ and practice nurses $(n=12)$ from the 'GP Access' Division of General Practice between March and July 2007.

Results: General practitioners' relationships with dietitians was believed to be the primary influencing factor on referral, provided by $81 \%$ of Survey Dietitians and $25 \%$ of Interview Dietitians. The most common means of initially forming relationships with general practitioners were face to face introduction (48\%) and introductory letters $(37 \%)$. Patient feedback via letter, fax or email was the most popular method of maintaining relationships with general practitioners (77\%). Meeting with general practitioners in person was believed to be the most effective activity in building relationships with general practitioners and increasing referral rates (42\%). Referral was made easier for general practitioners by providing paper $(37 \%)$ or electronic $(19 \%)$ referral forms and contact details (19\%). The majority of general practitioners and dietitians believed that the 'Allied Health Services Under Medicare' made it easier to refer to a dietitian. 
Conclusions: Dietetic referrals can be encouraged through good relationships between general practitioners, practice nurses and dietitians. Private practice dietitians would benefit from cultivating relationships with general practitioners and practice nurses through personal contact and providing good patient feedback.

Keywords: dietetics, general practice, general practitioner, practice nurse, private practice, referral and consultation, relationships

\section{INTRODUCTION}

While general practitioners (GPs) and practice nurses (PNs) have a role in providing nutrition advice in general practice, they cannot replace dietitians' expertise. ${ }^{1,2}$ It is recommended by The American Dietetics Association as well as various health professionals that GPs screen their patients for nutrition-related conditions and provide the initial advice, then refer patients requiring additional individualised assistance to a dietitian. ${ }^{1-4}$ It is then necessary that GPs reinforce the importance of the nutrition advice provided by dietitians. ${ }^{3,5}$ Evidence also shows that PNs have an increasing role in advising patients about dietary behaviours. ${ }^{6,7}$

Within the past seven years Government initiatives ${ }^{8}$ have been introduced to improve access to private allied health professionals. The Medicare Chronic Disease Management (CDM) Program's Individual and Group Allied Health services under Medicare (previously part of the Enhanced Primary Care Program) are avenues by which patients with chronic disease can access nutrition advice provided by dietitians in private practice. ${ }^{8}$ While free allied health services are available to patients through the public health system, generally waiting periods are extensive. Medicare rebates improve the cost-effectiveness of the private system for patients, allowing for more timely access to services. The Government recognises the gate keeping role of GPs, with access to Medicare rebates being reliant on GP referral. ${ }^{8}$ 
There is an acknowledged benefit of dietetic services being located within the general practice setting in improving patient care through better patient access and uptake of services..$^{9,10}$ Location of dietetic services within general practice can improve dietitians' relationships with GPs and are convenient for patients. ${ }^{9-11}$ It is thought that improving collaboration between GPs and dietitians, made easier by co-location, will encourage referrals and assist in maximising patients' health. ${ }^{12}$

While dietitians are vital members of the primary health care team, unless referral occurs the role of dietitians in the general practice setting can not be utilised to its full potential. Splett's model of 'the cascade of events leading to evidence on the effectiveness and cost-effectiveness of nutrition interventions' ${ }^{13}$ was used as the theoretical framework that guided this study. This model can be used to evaluate systems issues and identify where the barriers exist for effective nutrition care. The aim of this article is to: examine dietitians' relationships with GPs and PNs as a factor that influences dietetic referrals.

\section{METHODS}

A mixed-methods approach was used which was primarily qualitative with a quantitative component (Figure 1). This involved semi-structured telephone interviews and an online survey with PP (private practice) dietitians as well as questionnaires with GPs and PNs. The mixed methods approach allowed for evaluation of the topic from a variety of perspectives. This post hoc study was developed as a result of the poor delivery of nutrition advice by GPs and PNs as part of a 'Lifescripts' model. Questionnaires were self-completed by GPs and PNs from a Division of General Practice (DGP) in the Hunter region of NSW, Australia, between March and July 2007 (phase 1). This division was selected because of its physical proximity to the researchers. GP members of the Health Evaluation and Research Network $(n=18)$ were sent information statements twice. A recruitment advertisement was also placed in the division newsletter. GPs from a practice with an on-site dietitian were also recruited. 
PNs were recruited via a mailed information package sent to all practices who had previously expressed interest in Lifescripts (Available from: http://www.health.gov.au/lifescripts, accessed 18 January 2012) within the same DGP $(n=73)$. Therefore, these participants are likely to present a 'best case' scenario rather than a representative sample.

Semi-structured telephone interviews with PP dietitians (Interview Dietitians) were conducted from April to May 2008. PP dietitians included those providing services while working in the private capacity, as per the Medicare provider eligibility. ${ }^{14}$ The interviews explored participants' opinions of the provision of nutrition advice in general practice including 'Allied Health Services Under Medicare', GP referral and participants relationships with GPs. Participants were PP dietitians in Australia working within a selection of Divisions of General Practice (DGP) deemed to have a high or low number of dietitian Chronic Disease Management (CDM) consultations per population ${ }^{15}$ and/or PP dietitian full-time equivalents (FTEs). There were no exclusion criteria. Divisions providing either a high or low relative number of CDM consultations were selected to evaluate whether differences in dietitians' opinions or behaviours existed that accounted for the differing provision of CDM consultations. The number of dietitian CDM consultations per DGP was obtained from Medicare Australia. PP dietitian FTEs per division were calculated from the Dietitians Association of Australia (DAA) 2007 membership data using postcodes. Divisions were ranked by provision of $\mathrm{CDM}$ dietetics consultations and the highest and lowest ranked divisions were selected based on urban or rural status, in order to attain a broad range of eligible participants. Eighteen divisions were chosen, eight high CDM (H- CDM) and ten low (L- CDM) as this is where the natural separation of groups occurred. Divisions with fewer than one FTE were excluded with the exception of one Tasmanian division as all three Tasmanian divisions were in this category. More urban divisions were selected to reflect their greater representation in Australia (11 urban; 7 rural). 
The 'Find an APD' (Accredited Practicing Dietitian) online database ${ }^{16}$ provided by DAA was used to obtain a list of dietitians who reported working in each division. Postcodes included in each of the DGP were sourced from the Australian General Practice Network 'Network Directory' ${ }^{17}$ Eligible dietitians were invited to participate via email/mail. Reminder emails were sent to non-respondents after three weeks. Interviews took on average 15-25 minutes, and were recorded with participant permission. The interview consisted of 40 questions which covered: demographic data; strategies to increase the delivery of nutrition advice; the provision of nutrition advice by GPs and PNs; participants' involvement in, and opinions of 'Allied Health Services Under Medicare'; GP referral, including strategies used to initiate, maintain and assist in referral; and participants relationships with GPs. Questions were developed from literature ${ }^{18-20}$ and the GP/PN surveys, and pilot tested.

An online survey was conducted with PP dietitians (Survey Dietitians) from April to May 2008 (Phase 3). This assessed participants' opinions on the provision of nutrition advice in general practice, including the use of Lifescripts@ by GPs and PNs. Dietitians working in PP in Australia were invited to participate $(n=770)$. PP dietitians were recruited via email link to the online electronic quantitative survey using the "DAA weekly member email", a national communication tool for practitioners. Reminder invitations were sent via "DAA Private Practice Interest Group" email. The survey consisted of 31 questions. These were formulated based on interview questions and the literature ${ }^{18}$ and pilot tested. Response options consisted of open-, partially closed-, and closed-ended questions, including ordered and unordered response categories. ${ }^{21}$ As the online survey was anonymous, dietitians participating in telephone interviews were not excluded.

Approval for the projects was gained from the Human Research Ethics Committee at the University of (removed for blind review); approval numbers H-2008-0070; H-20080038; H-253-0706 and H-403-0407. 
Data analysis was conducted using Microsoft Excel 2003 and Intercooled Stata 9.1.22 Qualitative data was clustered into themes and tabulated. Quantitative data was tabulated, and counts and proportions used to summarise data and explore differences between groups.

\section{RESULTS}

Telephone interviews were conducted with 52 PP dietitians (22\% response rate); 18 dietitians from divisions providing a high number of CDM consultations (30\% response rate) and 37 providing a low number (20\% response rate); three participants worked in both a H-CDM and L-CDM division. At this point data saturation occurred. Ninety PP dietitians completed an online survey (12\% of 2007 DAA PP members). Compared to DAA 2007 membership data, Interview Dietitians reported working more hours than anticipated, while Survey Dietitians had graduated more recently; no other demographic differences were observed. Just over half of Interview and Survey Dietitians reported to work in GP surgeries (58\% and $52 \%$ respectively). Questionnaires were conducted with 11 GPs and 12 PNs.

\begin{tabular}{|c|c|c|c|c|}
\hline & \multicolumn{2}{|c|}{$\begin{array}{c}\text { Interview } \\
\text { Participants } \\
(n=52)\end{array}$} & \multicolumn{2}{|c|}{$\begin{array}{c}\text { Survey } \\
\text { Participants } \\
(n=90)\end{array}$} \\
\hline & $\mathrm{n}$ & $(\%)$ & $\mathrm{n}$ & $(\%)$ \\
\hline \multicolumn{5}{|l|}{ Gender } \\
\hline Female & 50 & $(96)$ & 80 & $(91)$ \\
\hline \multicolumn{5}{|l|}{ Practice locations } \\
\hline GP surgery & 30 & (58) & 47 & $(52)$ \\
\hline With other Allied Health Professionals & 14 & (27) & 30 & (33) \\
\hline Own office & 14 & (27) & 15 & (7) \\
\hline Specialist centre & 9 & (17) & 5 & (a) $(6)$ \\
\hline Home office & 6 & $(12)$ & 11 & $(12)$ \\
\hline Sports clinic/gym/club rooms & 5 & (10) & 5 & (6) \\
\hline Nursing home & 3 & (6) & 5 & (6) \\
\hline Private hospital & 2 & (4) & 5 & (6) \\
\hline Home visits & 2 & (4) & 18 & (a) $(20)$ \\
\hline Private rooms in hospital complex & 1 & $(2)$ & 1 & (1) \\
\hline
\end{tabular}


The majority of Interview Dietitians believed they had positive relationships with the GPs they worked with (64\%), while 25\% either reported good relationships with only some of the GPs or average relationships. Poor relationships were reported by $12 \%$, including many who were mainly reliant on specialist referral, including paediatricians, endocrinologists and gastroenterologists.

A GPs' relationship with a dietitian was believed to be the main influencing factor for referral by $81 \%$ of Survey Dietitians (tick box responses) and $25 \%$ of Interview Dietitians (open ended responses). They also believed that a dietitian knowing the GP enhanced the likelihood of referral (56\% and 15\% respectively): 'GPs need to know the person they're referring to. They do not particularly want to refer their patients to someone they have never met.' (Interview 3)

Table 2 indicates the reported strategies used by Interview Dietitians to initially form and maintain relationships with GPs. The most common means of initially forming relationships with GPs were face to face introduction (48\%), introductory letters (37\%) and patient feedback letters (25\%). H-CDM Participants reported they were more likely to have formed initial relationships with GPs via their involvement in the division $(11 \%$ vs. $0 \%$; $=0.039)$ or GPs already referring to their practice before they started working there $(11 \%$ vs. $0 \%$; $\mathrm{p}=0.039)$.

The strategies used by Interview Dietitians to maintain relationships with GPs are provided in Table 2. Patient feedback via letter, fax or email was the most popular method of maintaining relationships with GPs (77\%), followed by phone calls $(25 \%)$ and good communication and contact (23\%) (including feedback that was: timely, succinct, informative, consistent and not generic). No significant differences in 
methods of maintaining relationships with GPs were observed between H-CDM and LCDM Interview Dietitians.

Table 3 presents the strategies used by Interview Dietitians to make referral easier for GPs. The most commonly reported strategy was to make referral easier for GPs by providing paper referral forms (37\%). However $37 \%$ of those mentioning paper referral forms reported that they were not effective or they no longer used them due to the increased use of electronic systems. Electronic referral forms either via a website or software were reported by $19 \%$. Providing contact details to GPs was mentioned by $19 \%$, either in the form of business cards (12\%), information letters/brochures $(8 \%)$, appointment cards $(2 \%)$ or letter heads (2\%). L-CDM Participants reported higher rates of making referral easier for GPs by explaining the process of referral or demonstrating how to do the paperwork ( $19 \%$ vs. $0 \%$; $\mathrm{p}=0.048)$.

Meeting GPs in person was believed to be the most effective activity in building relationships with GPs and increasing referral rates (42\%): 'the [GPs] who see her more often are the ones who more often refer. If you're out of sight you're out of mind' (Interview 11). It was also identified that personally explaining their service and what they do with patients was effective:

Meeting with the practices and explaining our services and how they can complement the EPC $[\mathrm{CDM}]$ services of the GP. Also showing the practices what we actually do with individual clients when they come in, what sort of process we would undertake in each appointment and what the clients will get from attending an appointment... Some GPs probably aren't really aware of exactly what would happen in a dietetic consultation, and once they see what we do they are usually quite surprised. (Interview 18)

Providing GPs with good feedback was believed to be the second most effective activity in building relationships and increasing referrals (39\%). This included phone calls, good report writing and timely feedback: 'The quality of the doctors letters that I send back, and how quickly they come back' (Interview 24). Other activities that were commonly reported to be effective were: good outcomes with patients (15\%); 
information/updates on service (14\%); talks/education sessions (12\%); and good contact/communication (12\%).

More than half of the PP dietitians worked in a GP surgery (Interview: 58\%; Survey: $52 \%)$. The majority of GPs $(n=8 / 10)$ and PNs $(n=8 / 12)$ agreed that having a dietitian within the practice would make it easier to refer, as did Interview Dietitians (69\%). The main reasons suggested was that it acted as a reminder or provided better exposure (31\%): 'I believe 'in sight in mind'...being in there with them, working with them, seeing them regularly, that's the best way to go' (Interview 6). Better communication and interaction was reported by $29 \%$ : You 'actually get to talk to the GPs as opposed to never being able to see them' (Interview 5). It was also to provide easier access for patients (23\%): 'You'd be right there on the spot and the client would be more inclined to make an appointment with you before they left the surgery' (Interview 8). Better relationships between GPs and dietitians or the GPs knowing the dietitians were suggested by $16 \%$. The main reason suggested for co-location being a deterrent to referral was that it was only easier for the GPs in that particular practice (10\%): 'I think other GPs in different practices tend to generally very hesitant to refer patients to see you if you're working in a different doctor's room.' (Interview 4)

\section{DISCUSSION}

Good relationships between GPs and dietitians have been shown to be important in influencing GPs' referral rates.9, 23, 24 It is therefore important to understand dietitians' relationships with GPs, how they were forged and how the nature of the relationship and perceived barriers impact on referral. In this study the majority of Interview Dietitians reported positive relationships with the GPs with whom they worked. A variety of strategies to initiate and maintain relationships with GPs including face to face contact and providing good feedback were believed to be the most effective. Research conducted on behalf of the DAA showed that GPs desire face-to-face contact 
and thorough feedback. ${ }^{24}$ Similarly, the literature identifies that adequate and timely communication is necessary for effective collaboration. ${ }^{12,23}$

Communication was assisted with the use of referral forms, both paper and electronic. These were the most common strategy to ease the referral process for GPs. Providing contact details in the form of business or appointment cards, brochures or information on letterhead were also useful. These appear logical strategies for improving referral rates for dietitians; however, there is no evidence pertaining to this in the literature. Changing technology appears to be impacting on the referral process, with an increased use of an online environment.

Dietitians providing details about their services and clarifying processes to GPs was reported by participants as an effective way to support good relationships. This would overcome many of the primary barriers to GPs referring indentified in the literature, including a lack of knowledge of the service provided, the GPs' opinion of the skills of a dietitian or the knowledge of who to refer.9, 20,25 Increasing GPs' awareness of a nutrition service was also shown to increase referral rates. ${ }^{26}$ Many GPs are not aware of the role of a dietitian, and therefore outlining the patient experience of the dietitian's service may increase GPs' awareness and confidence in the dietitian, thus increase referrals.

There was agreement amongst the GPs, PNs and dietitians that co-location at the GP surgery would improve referral rates. Dietitians believed being located in a GP surgery made it easier for GPs to refer to them; however, it was acknowledged that it then may reduce referrals from GPs outside of the particular practice. Co-location with GPs leads to easier and improved communication ${ }^{10,11}$ which can lead to a greater recognition of being a vital member of the general practice team. ${ }^{9,11}$ Dietitian and GP co-location was also acknowledged to be more convenient for patients." ${ }^{91}$ A GP surgery was the most common practice location for Interview and Survey Dietitians with more than half of 
the participants working in this location. Contrary to this, research by Cant and Aroni (2008) show that the most common practice location for their participants was sole practice (49\%), followed by a GP practice ( $29 \%$ of participants). ${ }^{27}$ The higher responses of dietitians who work in GP practices in this research may have been due to the increased interest in the area of nutrition advice in general practice.

The quality and characteristics of relationships with GPs was perceived to be similar between H-CDM and L-CDM dietitians suggesting that this does not impact on the number of $\mathrm{CDM}$ referrals provided. $\mathrm{H}-\mathrm{CDM}$ participants were more likely to have initially formed relationships with GPs via their involvement in the DGP suggesting division support may be useful in obtaining Medicare referrals. They were also more likely to report that initial relationships with GPs were formed prior to their involvement in the practice, indicating larger practices that may have effective Medicare referral processes established. Differences between H-CDM and L-CDM participants do not indicate which strategies to maintain relationships or make referral easier are more effective.

While there are small participant numbers in the responses from GPs and PNs, their comments make a valid contribution to this body of literature, however, they may not be representative to the GP and PN population across Australia more generally. As these GPs and PNs are likely to have a greater interest in nutrition they may represent a 'best case' scenario. The use of two forums to gain information from the dietitians, the telephone interview (open ended questions) and the online tick box questionnaire, mean that interpreting the responses must be done with caution due to the differing levels of prompting provided. Additionally, interview and survey tools were not validated and PP dietitians may have participated in both the telephone interview and online survey.

Dietitians are the acknowledged experts in nutrition and are vital members of the primary health care team. However, access for patients who require individualised 
nutrition advice from PP dietitians is often limited due to poor rates of referral. It is clear that dietitians' relationships with general practice staff is a key factor that influences dietetic referrals. Dietitians who wish to enhance the opportunity for patients to receive specialised nutrition services need to be proactive. They need to ensure that relationships with primary providers are robust and that time and resources are available to ensure that good feedback is provided in a timely and efficient manner. Co-location is one way that relationships can be enhanced, but is not deemed essential. Other forms of personal communication can also be effective such as telephone and email contact. There are inconsistent reports from participants regarding the value of new technologies; however they may contribute to strengthening communication and hence relationships, if used well. There is a need for PP dietitians to understand these systems and to accept responsibility for relationship building if they hope to increase access and referrals to their services.

\section{REFERENCES}

1 Macario E, Emmons KM, Sorensen G, Hunt MK, Rudd RE. Factors Influencing Nutrition Education for Patients with Low Literacy Skills. J Am Diet Assoc. 1998; 98: 559-64.

2 American Dietetic Association. Position of The American Dietetic Association: Nutrition education for health care professionals. J Am Diet Assoc. 1998; 98: 343-6.

3 Brauer P, Dietrich L, Davidson B. Nutrition in Primary Health Care: Using a Delphi Process To Design New Interdisciplinary Services. Can J Diet Pract Res. 2006: S14.

4 Pomeroy SE, Worsley A. Enhancing the dietary management of general practice patients through collaborative care: perspectives of general practitioners and dietitians. Nutrition \& Dietetics. 2009; 66: 81-6.

5 Truswell AS, Hiddink GJ, Blom J. Nutrition guidance by family doctors in a changing world: problems, opportunities, and future possibilities. American Journal of Clinical Nutrition. 2003; 77: 1089S-92.

6 Pineiro R, Brotons C, Bulc M, et al. Healthy diet in primary care: views of general practitioners and nurses from Europe. Eur J Clin Nutr. 2005; 59: S77-S80.

$7 \quad$ Britt H, Miller GC, Charles J, et al. General practice activity in Australia 200506. AIHW Cat No GEP 19. Canberra:: Australian Institute of Health and Welfare, 2007. 
8 Department of Health \& Ageing. MBS Primary Care Items: Individual Allied Health Services for patients with a chronic medical condition and complex care needs under $\quad$ Medicare. 2010.2 (Also available from: http://www.health.gov.au/internet/main/publishing.nsf/Content/mbsprimarycarechronicdiseasemanagement, accessed 2nd July 2010).

9 Lowe S, Lawrence R. Allied Health and the medical workforce - teamwork, integration and sustainability. 8th National Rural Health Conference, 2005.

10 Witt J, Brauer P, Dietrich L, Davidson B. Estimation of Human Resource Needs And Cost of Adding Registered Dietitians To Primary Care Networks. Can J Diet Pract Res. 2006; 67: S30-8.

11 Bradshaw C. Does the employment of an on-site dietitian improve the uptake of dietetic care in general practice diabetes clinics? Practical Diabetes. 1994; 11: 213-4.

12 Hurley C, Kalucy E, Battersby M. General practitioners' collaboration with service coordinators: what makes it work? Lessons from SA HealthPlus Coordinated Care Trial. Australian Journal of Primary Health. 2002; 8: 45-51.

13 Splett. Cost Outcomes of Nutrition Intervention: Part 1 of a 3-Part Monograph: Mead Johnson \& Company, 1996.

14 Medicare Australia. Application for an initial Medicare provider/registration number for an Allied Health Professional. 2006. (Also available from: http://www.medicareaustralia.gov.au/provider/pubs/medicare-

forms/files/ma_1449_app_for_initial_medicare_provider_registration_number_for_allie d_health_professional.pdf, accessed 30th March 2011).

15 Primary Health Care Research \& Information Service. Key Division of General Practice Characteristics 2004-2005. 2006.

16 Dietitians Association of Australia. Find a Consulting APD. Canberra; 2010. (Also available from: http://www.daa.asn.au/dmsweb/frmfindapdsearch.aspx, accessed 4th July 2010).

17 Australian Divisions of General Practice. Australian Divisions of General Practice Network Directory. 2006. (Also available from: http://www.adgp.com.au/site/index.cfm?display=301, accessed Aug 2006 2006).

18 Cant R, Aroni R. Melbourne dietitians' experience of Medicare policy on allied health services (Strengthening Medicare; Enhanced Primary Care) in the first 12 months. Nutrition \& Dietetics. 2007; 64: 43-9.

19 Sims J, Tacticos T, Potiriadis M, Naccarella L. Evaluation of a program to assist usage of the Enhanced Primary Care case conferencing and care planning items: the allied health professionals' viewpoint. Australian Journal of Primary Health. 2004; 10: 72-7.

20 Nicholas LG, Pond CD, Roberts DC. Dietitian-general practitioner interface: a pilot study on what influences the provision of effective nutrition management. American Journal of Clinical Nutrition. 2003; 77: 1039S-42S.

21 Dillman DA. Mail and Internet Surveys: The tailored design method. Hoboken, New Jersey: John Wiley \& Sons, 2007. 
22 StataCorp. Stata Statistical Software: Release 9.0. In: College Station, editor. TX, 2005.

23 Splett PL, Reinhardt MA, Fleming P. Physicians' expectations for quality nutrition expertise and service in prenatal care. J Am Diet Assoc. 1994; 94: 1375-80.

24 Dietitians Association of Australia. DAA invests in GP research to help members increase referrals. DAA Newsletter. 2009: 17-8.

25 Kelly A, Joffres MR. A survey of physicians' uses/opinions regarding nutrition education resources. J Can Diet Assoc. 1990; 51: 409-12.

26 Pediani L, Bowie P. The benefits of dietitian-led community clinics for people with diabetes: using audit to raise GP awareness. Practical Diabetes International. 1999; 16: 9-11.

27 Cant R, Aroni R. Simplifying the mechanics of patient care under Medicare for dietitians in private practice; an evaluation of dietetics Medicare services for Chronic Disease Management. Clayton, Victoria, Australia: Monash University: Monash Institute of Health Services Research, 2008. 
PHASE 1

GPs \& PNs -

Implementation of Lifescripts $(\subset)$ \& questionnaires

GPs and PNs' opinions and behaviours regarding:

- $\quad$ use of Lifescripts@

- delivery of nutrition advice

- $\quad$ referral to dietitians

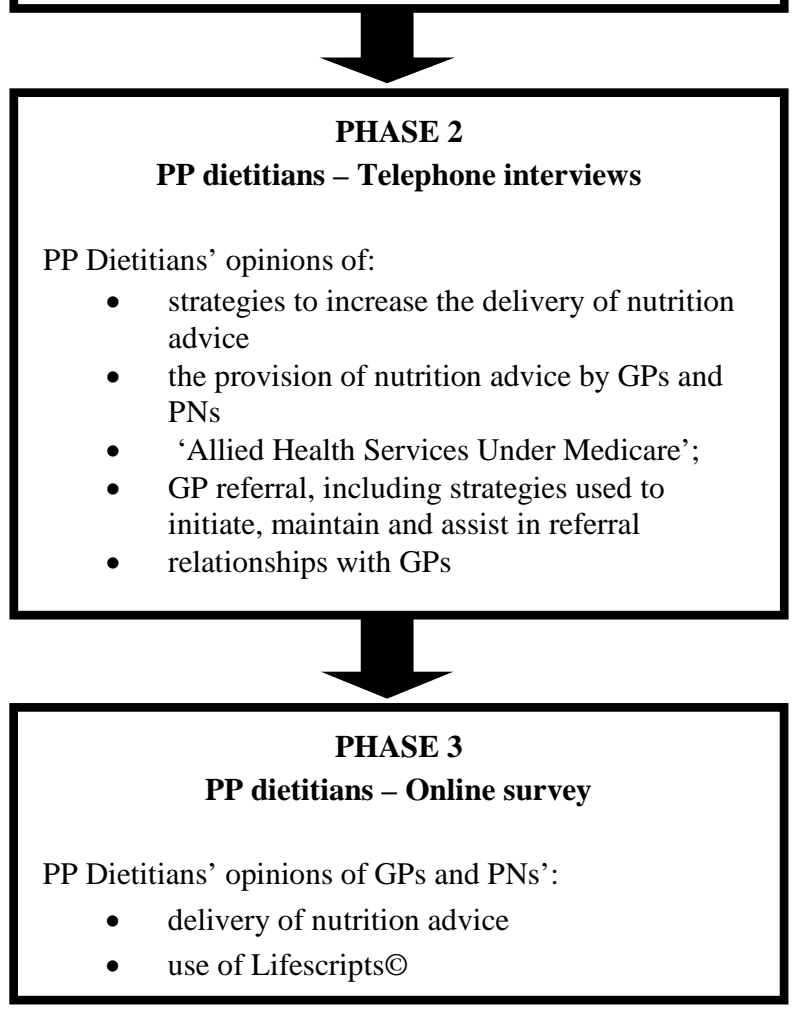

2 Figure 1 Overview of the mixed-methods research design

$3 \mathrm{GP}=$ General Practitioner; $\mathrm{PN}=$ Practice Nurse; $\mathrm{PP}=$ Private Practice 
1 Table 1 Strategies used by Interview Dietitians to initially form and maintain 2 relationships with general practitioners

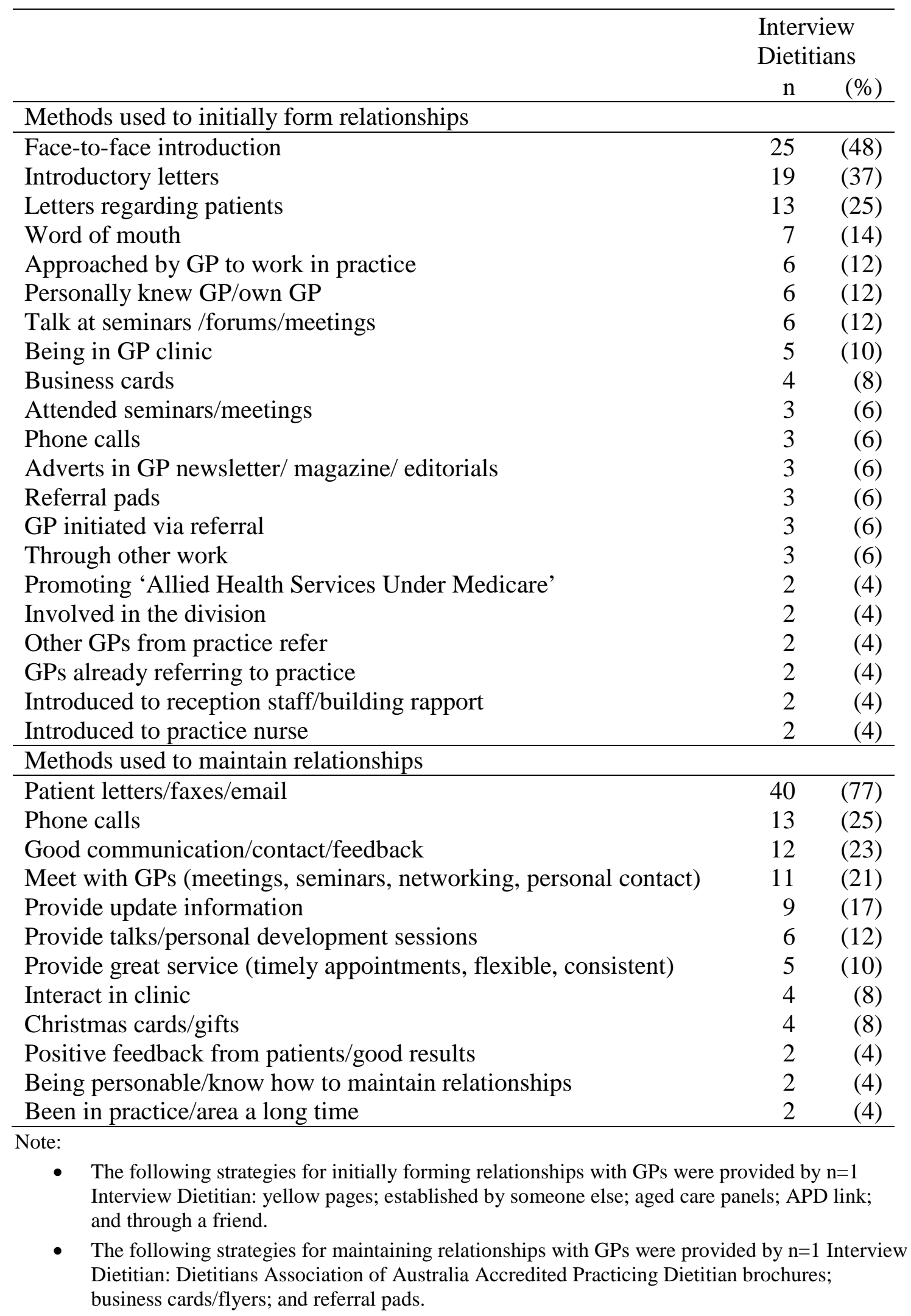


1 Table 2 Strategies used by Interview Dietitians to make referral easier for GPs

\begin{tabular}{|c|c|c|}
\hline & \multicolumn{2}{|c|}{$\begin{array}{l}\text { Interview } \\
\text { Dietitians }\end{array}$} \\
\hline & $\mathrm{n}$ & $(\%)$ \\
\hline Referral forms/pad & 19 & (37) \\
\hline $\begin{array}{l}\text { Electronic referral (form on website/contact details in } \\
\text { computer/email referrals) }\end{array}$ & 10 & (19) \\
\hline $\begin{array}{l}\text { Provided contact details (business/appointment cards/ } \\
\text { brochures/information on letter head) }\end{array}$ & 10 & (19) \\
\hline Explained process of referral to GPs/how to do paperwork & 7 & (14) \\
\hline $\begin{array}{l}\text { Provided details about service (to GPs/practice nurse and patient } \\
\text { brochures) }\end{array}$ & 6 & $(12)$ \\
\hline Promote Medicare service and that they are a provider & 5 & $(10)$ \\
\hline Located in practice & 4 & $(8)$ \\
\hline Being contactable (receptionist available at all times/phone directly) & 4 & (8) \\
\hline $\begin{array}{l}\text { Advertising conditions that can be referred/areas of practice/ } \\
\text { specialising }\end{array}$ & 3 & (6) \\
\hline Practice in convenient location & 3 & (6) \\
\hline Fax/phone referral & 2 & (4) \\
\hline On Division of General Practice lists & 2 & (4) \\
\hline Bulk bill/provide 'Allied Health Services Under Medicare’ & 2 & (4) \\
\hline Feedback/communication & 2 & (4) \\
\hline
\end{tabular}

2 Note: the following strategies were provided by n=1 Interview Dietitian: Dietitians Association of 3 Australia ‘Advanced Dietitian’ brochures; business cards/flyers; and referral pads. 